\title{
Estudio de variables asociadas a la psicoterapia grupal en los procesos de duelo patológico.
}

Study of variables associated to the group psychotherapy in the process of pathological grief.

\author{
Juan Díaz Curiel a. \\ ${ }^{a}$ Psicólogo Clínico del Servicio de Salud Mental de Vallecas Villa de Madrid.
}

Correspondencia: Juan Díaz Curiel (juandcsj@gmail.com)

Recibido: 13/04/2010; aceptado con modificaciones: 1/09/2010

\begin{abstract}
RESUMEN: Se estudian variables asociadas al proceso terapéutico grupal (10 sesiones de 75 minutos) de 24 sujetos adultos (23 mujeres y 1 varón) distribuídos en cuatro grupos de terapia durante los años 2007-2010 con diagnóstico de duelo complicado (DC). El DC se evalúa a través de la Escala de Prigerson (punto de corte >24) (Prigerson, 1995, Traducción Española Olmeda y García, 2006). Los pacientes pertenecen al Equipo de Salud (ESM) de Vallecas Villa y acuden remitidos por el médico de Atención Primaria para evaluar y tratar síntomas compatibles con duelo complicado. En otros casos el DC se detecta en las primeras entrevistas de evaluación, o bien a lo largo del tratamiento psiquiátrico o psicoterapéutico por los profesionales del ESM. Una vez finalizado el tratamiento grupal (TG) se vuelve a pasar La Escala de Prigerson obteniendo como resultado diferencias significativas en las puntuaciones antes y después del tratamiento, indicador de que el duelo complicado mejora significativamente en los sujetos que han participado en el tratamiento grupal. PALABRAS CLAVE: Psicoterapia de Grupo. Duelo Patológico
\end{abstract}

\begin{abstract}
This paper presents the study of variables associated to the group treatment (10 sessions of 75 minutes) of 24 adult subjects (23 females and 1 male) divided into four therapy groups during the years 2007-2009 with a diagnosis of complicated grief (DC), evaluated through Prigerson Scale (cutoff> 24) (Prigerson, 1995, Spanish Translation Olmeda and García, 2006), belonging to the Mental Health Team Vallecas Villa (ESM). The patients were sent by their primary care physician to assess and treat symptoms consistent with complicated grief; in other cases the DC was detected in the early evaluation interviews, or along the psychiatric or psychotherapeutic treatment by professionals from ESM. Once finalized the group treatment (TG) becomes to pass the Scale of Prigerson obtaining results statistically differents in the scores, indicated that complicated grief improvement significantly in the subjects that have received group psycotherapy.

KEY WORDS: Psychotherapy, Group. Pathological Grief.
\end{abstract}

"Un día hay vida. Por ejemplo, un hombre de excelente salud, ni siquiera viejo, sin ninguna enfermedad previa. Todo es como era, como será siempre. Pasa un día y otro, ocupándose sólo de sus asuntos y soñando con la vida que le queda por delante. $Y$, entonces, de repente, aparece la muerte”

La Invención de la Soledad. Paul Auster.

\section{Introducción}

Se presentan los resultados del estudio de variables asociadas al tratamiento grupal (10 sesiones de 75 minutos) de 24 sujetos adultos (23 mujeres y 1 varón) distribuidos en cuatro grupos de terapia con diagnóstico de duelo complicado (DC) a través de la Escala de Prigerson (1) (2) (Anexo 1) (punto de corte >24) (adaptación Española Olmeda y García, 2006), pertenecientes al Equipo de Salud Mental de Va- 
llecas Villa durante los años 2007-2010. Los pacientes acuden remitidos por el médico de Atención Primaria para evaluar y tratar síntomas compatibles con duelo complicado, en otros casos el DC se detecta en las primeras entrevistas de evaluación, o bien a lo largo del tratamiento psiquiátrico o psicoterapéutico por los profesionales del ESM.

La palabra "duelo" tiene sus raíces en el término latino "dolos" que significa dolor. El DSM-IV-TR incluye el duelo con el código V62.82 no atribuible a trastorno mental y lo define como la reacción ante la muerte de una persona querida. La CIE-10 emplea el código Z63.4 para referirse al duelo normal (desaparición o fallecimiento de un miembro de la familia) dentro de los factores que influyen en el estado de salud y en el contacto con los servicios de salud, problemas relacionados con el grupo de apoyo, incluidas las relaciones familiares e incluye en los Trastornos de Adaptación (F43.2) a las reacciones de duelo de cualquier duración que se consideren anormales para sus manifestaciones o contenidos.

En la actualidad el DC no está incluido en la Clasificación del DSM-IV-TR debido a la falta de consenso para su definición. Algunos de los síntomas que agravan el duelo normal y se acercan al duelo patológico (Complicado, traumático, crónico...) son los siguientes (3):

1. Culpa por las cosas, más que por las acciones

2. Pensamientos de muerte más que voluntad de vivir

3. Preocupación mórbida con sentimientos de inutilidad

4. Enlentecimiento psicomotor acusado.

5. Deterioro funcional acusado y prolongado

6. Experiencias alucinatorias distintas de las de escuchar la voz o ver la imagen fugaz de la persona fallecida.

Uno de los investigadores que más se han aproximado a la delimitación y definición del Duelo Complicado es Prigerson (1) que diferencia la "pena traumática" con presencia de síntomas de malestar por la pérdida (pensamientos intrusivos sobre la persona fallecida, añoranza, búsqueda del fallecido y soledad como resultado del fallecimiento) de los síntomas de "malestar traumático" (falta de metas y/o inutilidad respecto al futuro, sensación subjetiva de indiferencia o ausencia de respuesta emocional, dificultades para aceptar la muerte, excesiva irritabilidad, amargura y/o enfado en relación a la muerte). Es importante diferenciar el DC de otros diagnósticos y patologías recogidos en el DSM-IV-TR como el Trastorno por Estrés Postraumático, Trastorno Depresivo Mayor y Trastorno Adaptativo. En la clínica los duelos complicados pueden presentarse de las siguientes formas (4): 1) Congelado. 2) Paranoide. 3) Maniaco. 4) Depresivo. 5) Ansioso. 6) Somatizador. 7) Histeroide. 8) Obsesivo. 9) Con abuso de drogas o alcohol. 10) Actuador. 11) Otras formas psicopatológicas.

¿Cuándo debe tratarse un duelo? Por lo general los duelos no se tratan sino que se acompañan. En la mayor parte de los casos la función del médico, amigo, 
ORIGINALES Y REVISIONES

familiar es la de acompañar y compartir sentimientos dolorosos por la pérdida. La psicoterapia específica y reglada en los procesos de duelo se debe aplicar en los casos siguientes referidos por Worden (5): a) El duelo complicado se manifiesta como un duelo prolongado; b) El duelo se manifiesta a través de algún síntoma somático o conductual enmascarado y c) El duelo se manifiesta con una reacción exagerada.

Elegimos la modalidad de Terapia de grupo de tiempo limitado, ya que permite examinar y evaluar el duelo complicado dentro de una óptica de encuentro y empatía con otros compañeros de grupo con sentimientos (enfado, culpa, soledad, tristeza, rabia...), recuerdos y síntomas semejantes a los propios a la par que se analizan nuevos vínculos (terapeuta, otros pacientes, el grupo mismo) durante el tiempo en el que se desarrolla la Terapia de Grupo. La TG tiene una función educativa en el sentido de aprender de una experiencia grupal donde el tiempo para resolver el duelo se enmarca en un tiempo personal en cada uno de los miembros del grupo donde hay que tomar una actitud activa (en el paciente y en los terapeutas) dentro del proceso de elaboración del duelo.

De los estudios que analizan la eficacia de los Grupos de Terapia destaca el meta-análisis realizado por Kato y Mann (6). De los 8 trabajos revisados solo uno de los estudios refleja resultados positivos y en 6 de ellos la intervención grupal no tiene ningún efecto y en otro solo se encuentras resultados levemente positivos. Los miembros de dichos grupos no son población clínica con un diagnostico claro de Duelo complicado y constituyen más grupos de apoyo que promueven actividades de índole social sin cambios cognitivos y/o reestructurantes de la sintomatología asociada al DC.

Entre los factores terapéuticos que se dan en los procesos terapéuticos grupales (Yalom) (7), definidos por Bloch y cols. (8) y revisados por Almenta y cols., (9) que pueden ayudar a la mejoría y elaboración del duelo complicado en nuestro marco terapéutico grupal señalamos los siguientes: Altruismo; Cohesión grupo; Universalidad; Aprendizaje interpersonal -Interiorización-; Aprendizaje interpersonal -Exteriorización-; Orientación; Verbalización; Emulación (Identificación); Reactivación de la experiencia familiar; Comprensión de sí mismo; Estimulación recíproca; Conciencia de la propia realidad.

Compartimos los principios asistenciales en el asesoramiento de duelos señalados por Worden y Tizón (4) (5)

1. Facilitar el afrontamiento de la realidad de la pérdida

2. Ayudar a identificar y expresar sentimientos (culpa, ira, ansiedad, triste$\mathrm{za}, \ldots)$

3. Ayudar a vivir sin el fallecido

4. Facilitar la reubicación del objeto interno

5. Dar tiempo

6. Interpretar la conducta normal 
7. Permitir las diferencias individuales

8. Ofrecer apoyo continuado no puntual, interrumpido o incierto

9. Ayudar a explorar lo adecuado o inadecuado de las defensas

10. Derivar si el duelo es patológico o muy complicado

Los objetivos generales del tratamiento grupal son: 1) Mejorar la sintomatología depresiva asociada a la pérdida de un familiar; 2) Mejoría de las relaciones socio-laborales y familiares; 3 ) Incrementar las Técnicas de afrontamiento frente a duelos en el futuro; 4) Incorporar el duelo como experiencia vital estresante de la que se puede salir reconfortado; 5) Reconocer y expresar con palabras los afectos y emociones estrechamente vinculados a la pérdida.

A los pacientes incluidos en la TG se les informa en entrevista individuales previas al inicio de los grupos de terapia sobre los siguientes objetivos: 1) Focalizar el contenido de las sesiones en el duelo 2) Tratar recuerdos, sentimientos y estados emocionales asociados a la persona fallecida. 3) Recuperar la capacidad de amar y disfrutar 4) Desplegar nuevas capacidades yoicas amenazadas y/o negadas por la persistencia del duelo patológico.

El encuadre grupal se organiza alrededor de las siguientes características: Grupo cerrado (máximo 10 personas); 10 sesiones de 75 minutos; Semanal. Día y horas prefijados. Terapeuta responsable grupo y coterapeuta observador; Asociación Libre (centrado en el duelo); Terapeuta activo, no intrusivo. Facilitador; Respeto, confidencialidad.; Asistencia obligada (excepto motivos de enfermedad u otros); Elaboración del duelo por fin de grupo.

Los pacientes acuden remitidos por el médico de Atención Primaria para evaluar y tratar síntomas compatibles con la elaboración patológica de un duelo, en otros casos el DC se detecta en las primeras entrevistas de evaluación de los pacientes o bien a lo largo del tratamiento psiquiátrico o psicoterapéutico por los profesionales del ESM. Una vez finalizado el tratamiento grupal se vuelve a pasar por los profesionales del Centro de Salud Mental la Escala de Prigerson con el objetivo de estudiar la variación de las puntuaciones antes y después del tratamiento y poder relacionar las mismas con variables socio-familiares, clínicas y del propio proceso psicoterapéutico grupal.

Los criterios de inclusión de los pacientes en los grupos de terapia son los siguientes: 1) Especificación en el PIC de duelo reciente (3-6 meses) como motivo de consulta; 2) Detección de la pérdida de un familiar como Acontecimiento Vital Estresante; 3) Conciencia por el paciente de que el duelo está vinculado con la situación de sufrimiento actual; 4) Entrevista de evaluación por parte del responsable del Grupo de Terapia previa inclusión al mismo. 5) Puntuación >24 en la Escala de Prigerson (realizada por alguno de los profesionales del Centro). Por el contrario se excluyen a: 1) Pacientes con sintomatología psicótica activa y/o diagnosticados de psicosis;2) 
ORIGINALES Y REVISIONES

Cuadros afectivos de depresión mayor; 3) Pacientes con negación masiva del duelo.

En la revisión de la literatura científica en nuestro entorno relacionada con el tratamientos grupal de los procesos de duelo patológico y/o traumático en los que se ha evaluado los resultados mediante escalas y pruebas psicológicas o bien técnicas de registro, se destaca la efectividad y eficacia de los mismos en aspectos como: 1) Elaboración de la pérdida y la adaptación a la nueva situación (10); 2) Mejoría en aspectos depresivos, mecanismos defensivos, relaciones interpersonales (11). Los tratamientos pueden ser en grupos cerrados (11), (12), (13), (14), (15) u abiertos (10), (16) de tiempo limitado entre 2-21 sesiones y 90 minutos de media de duración.

\section{Material y Método. Sujetos:}

Se seleccionan y estudian variables agrupadas en categorías descriptivas, socio-familiares y del proceso psicoterapéutico tal y como se presentan en la Tabla 1, correspondientes a 24 sujetos adultos ( 23 mujeres y 1 varón) distribuidos en cuatro grupos de terapia con diagnóstico de duelo complicado (DC) a través de la Escala de Prigerson (1) (2) (Anexo 1) (punto de corte >24) (adaptación Española Olmeda y García, 2006), pertenecientes al Equipo de Salud Mental de Vallecas Villa durante los años 2007-2010.

Para la evaluación del DC hemos utilizado: a) La Escala de Prigerson (1) que discrimina a sujetos con duelo normal y duelo patológico (punto de corte $>24$ ) y b) La Clínica del paciente extraída de la anamnesis y la entrevista de evaluación con los profesionales del Centro.

Se aplica un estudio estadístico del Programa SPSS para analizar y comparar las puntuaciones pre-tratamiento y postratamiento grupal de la Escala de Prigerson que diferencia el duelo normal del duelo complicado y planteamos la hipótesis de que esperamos encontrar diferencias significativas en las puntuaciones de las mismas antes y después del tratamiento grupal. Se eligen Estadísticos no Paramétricos debido al número pequeño de pacientes tratados, aunque la muestra y la puntuaciones responden a una distribución normal. Aplicamos la Prueba de Wilconson para comparación de medias. 
Tabla 1

Las religiones ante la muerte del feto.

\begin{tabular}{|l|l|l|}
\hline $\begin{array}{l}\text { VARIABLES PERSONALES } \\
\text { Y SOCIO-FAMILIARES }\end{array}$ & $\begin{array}{l}\text { VARIABLES RELACIONADAS } \\
\text { CON LA DEMANDA } \\
\text { Y EL DUELO }\end{array}$ & $\begin{array}{l}\text { VARIABLES RELACIONADAS } \\
\text { CON EL TRATAMIENTO } \\
\text { GRUPO }\end{array}$ \\
\hline Sexo & Derivación/procedencia & Tiempo muerte e inicio grupo \\
\hline Edad (Grupo de edad) & Derivación Psicólogo/Psiquiatra & $\begin{array}{l}\text { Puntuación Prigerson } \\
\text { (Antes tratamiento grupo) }\end{array}$ \\
\hline Estado civil & ¿Acude con medicación? & $\begin{array}{l}\text { Puntuación Prigerson } \\
\text { (Post tratamiento grupal) }\end{array}$ \\
\hline Tipo convivencia & Origen de la demanda & Inclusión Grupo de terapia \\
\hline Nivel educativo & Tipo demanda derivante & Adherencia terapéutica al grupo \\
\hline Situación laboral & Tipo de PIC & $\begin{array}{l}\text { Resultado del tratamiento } \\
\text { (al finalizar el grupo) }\end{array}$ \\
\hline & Impresión diagnóstica & \\
\hline & Tipo de tratamiento & \\
\hline & Tipo de medicación & \\
\hline & Tiempo de tratamiento individual & \\
\hline & Vínculo con la persona fallecida. & \\
\hline & Edad de la persona fallecida & \\
\hline & Tipo de muerte & \\
\hline & &
\end{tabular}

\section{Resultados}

Diagrama 1

Proceso y seguimiento del tratamiento grupal.

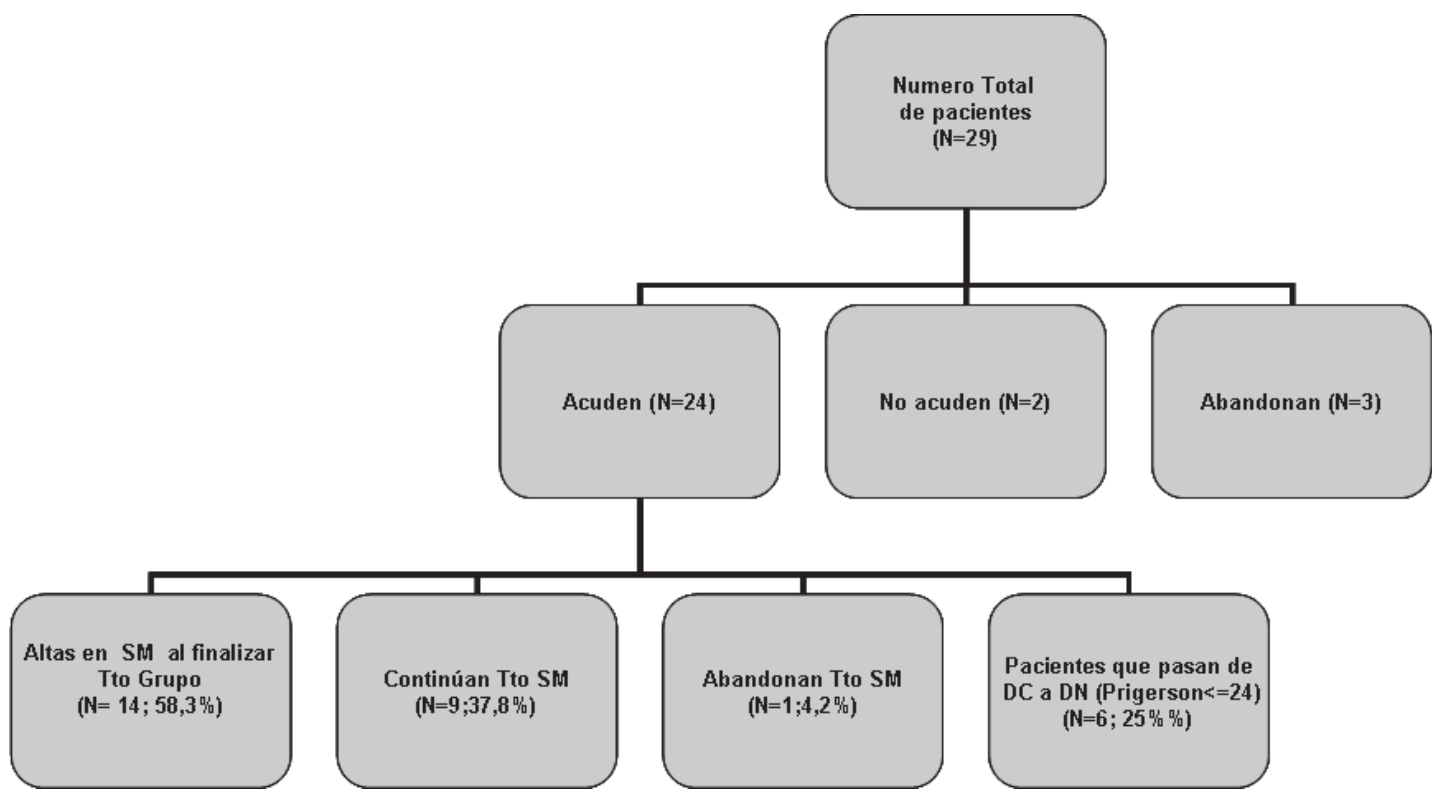


29 pacientes son derivados a terapia de grupo para tratar un posible Duelo Complicado y que cumplen el criterio de DC medido a través de la Escala de Prigerson (punto de corte $>24$ ). Dos sujetos no acudieron a la primera sesión de grupo y tres sujetos abandonaron durante las primeras sesiones de grupo. Al finalizar el tratamiento grupal, el 58,3\% son dados de alta en Salud Mental y el 37,5\% continúan en tratamiento psiquiátrico y/o psicoterapéutico (o combinado) en consultas de Salud Mental individuales. Al finalizar el tratamiento de grupo 6 sujetos (25\%) puntúan por debajo de 24 en la Escala de Prigerson, correspondiente a una puntuación de Duelo normal e indicador de una mejoría notable en el motivo por el por el que son tratados en la Terapia de Grupo para pacientes con Duelo Complicado.

Tabla 2

Variables descriptivas personales y socio-familiares de los pacientes.

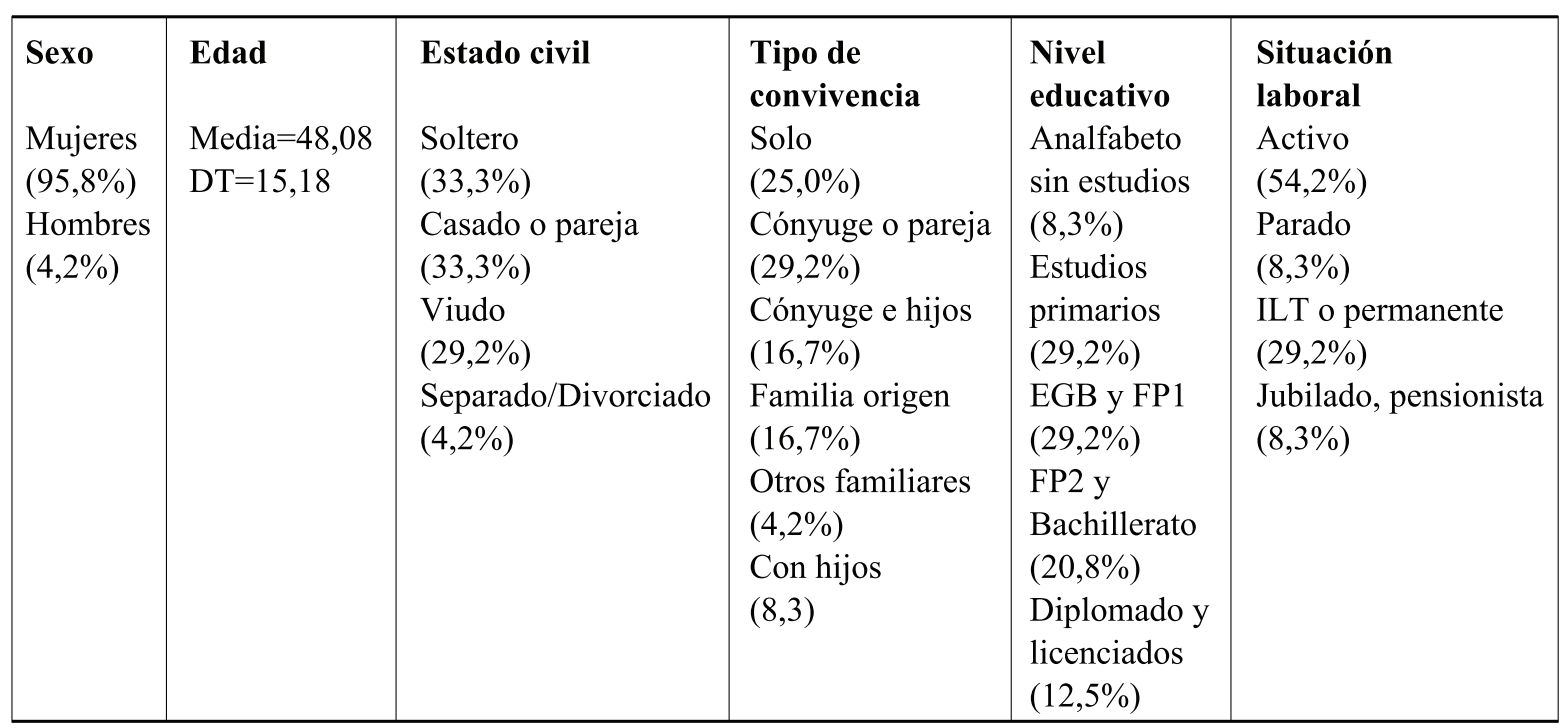

La gran mayoría de los pacientes que han acudido al grupo de terapia son mujeres, con una media de edad de 48 años, una tercera parte están casados y casi un $30 \%$ son viudos. Una cuarta parte viven solos. El nivel educativo es la formación básica (estudios primarios y EGB), destacando solo un 12,5\% con estudios superiores y activos laboralmente en su mayoría. 
Tabla 3

Variables clínicas relacionadas con la derivación y demanda

\begin{tabular}{|l|l|l|l|l|}
\hline Derivación & $\begin{array}{l}\text { Derivación a } \\
\text { salud mental } \\
\text { Médico }\end{array}$ & Medicación & $\begin{array}{l}\text { Origen } \\
\text { demanda }\end{array}$ & Tipo PIC \\
Cabecera & Psicuiatra $(41,7 \%)$ & Con medicación & A petición & Consulta nueva \\
$(91,7 \%)$ & Salud Mental (41,7\%) & $\begin{array}{l}\text { Sin medicación } \\
\text { propia }(25,0 \%)\end{array}$ & A petición & Consulta nueva \\
Otros $(8,3 \%)$ & & $(12,5 \%)$ & derivante & preferente $(37,5 \%)$ \\
& & & $(75,0 \%)$ & Revisión normal \\
& & & & $(4,2 \%)$ \\
& & & Sin PIC $(4,2 \%)$ \\
\hline
\end{tabular}

La práctica totalidad de los sujetos acuden remitidos a Salud Mental a petición del médico de familia en consultas nuevas normales o preferentes y llama la atención que en el 87,5\% de los casos ya con medicación.

Tabla 4

Variables clínicas relacionadas con el diagnóstico y el tratamiento en Salud Mental

\begin{tabular}{|l|l|l|l|}
\hline Diagnóstico & Tratamiento & Tipo medicación & Tratamiento individual \\
Trastorno & Psiquiátrico (58,3\%) & Ansiolíticos $(25,0 \%)$ & $1-3$ citas $(29,23 \%)$ \\
neurótico (45,8\%) & Consulta & AD+Ansiolíticos $(66,7 \%)$ & $4-6$ citas $(16,72 \%)$ \\
Trastorno Personalidad & psicoterapéutica $(25,0 \%)$ & AD $(4,2 \%)$ & $7-9$ citas $(20,8 \%)$ \\
$(12,5 \%)$ & Tratamiento combinado & Sin medicación $(4,2 \%)$ & $>10$ citas $(33,3 \%)$ \\
Trastorno afectivo (37,5\%) & $(16,7 \%)$ & & \\
Otros $(4,2 \%)$ & & & \\
\hline
\end{tabular}

La patología corresponde a trastornos afectivos y del espectro neurótico en su mayoría. El seguimiento en Salud Mental es mayoritariamente psiquiátrico (Antidepresivos+ ansiolíticos) o mixto psicoterapéutico y psiquiátrico. La cuarta parte acuden a consultas psicoterapéuticas con los psicólogos del Centro. Una tercera parte de los pacientes lleva ya tiempo en Salud Mental en tratamiento antes de empezar el tratamiento grupal (>10 citas)

Tabla 5

Variables relacionadas con características del duelo.

\begin{tabular}{|l|l|l|l|}
\hline Tipo de vínculo & Edad fallecido & Tipo de muerte & Tiempo transcurrido muerte \\
Padres $(66,7 \%)$ & $0-17$ años $(4,2 \%)$ & Enfermedad súbita $(33,3 \%)$ & $6-12$ meses $(58,3 \%)$ \\
Hijo $(12,5 \%)$ & $18-40$ años $(29,2 \%)$ & Enfermedad prolongada & $1-2$ años $(20,8 \%)$ \\
Pareja $(20,86 \%)$ & $41-65$ años $(16,7 \%)$ & $(58,3 \%)$ & $>2$ años $(20,8 \%)$ \\
& $>65(50,0 \%)$ & Accidente $(8,3 \%)$ & \\
\hline
\end{tabular}


La muerte de uno de los padres constituye el motivo principal del duelo complicado en nuestro grupo de pacientes $(66,7 \%)$ seguidos de la pareja y de un hijo. Por este motivo la mitad de las personas fallecidas supera los 65 años y el motivo mayoritario de causa de muerte es por enfermedades prolongadas, seguido por el de enfermedades súbitas. Para el 58,2 \% de los sujetos la muerte del familiar trascurre el año anterior al comienzo del tratamiento grupal.

Tabla 6

Variables estadísticamente significativas en la comparación de medias de la Escala de Prigerson antes y después del tratamiento.

\begin{tabular}{|c|c|}
\hline Variables estudiadas & $\mathbf{P}<0.05$ \\
\hline Puntuación Escala Prigerson & Antes y Después del Tratamiento Grupal $(\mathrm{p}=.001)$ \\
\hline Grupo de edad & $31-65$ años $(p=.002)$ \\
\hline Tipo convivencia & $\begin{array}{l}\text { Solo }(\mathrm{p}=.043) \\
\text { Cónyuge o pareja }(\mathfrak{p}=.034)\end{array}$ \\
\hline Tipo de muerte & $\begin{array}{l}\text { Enfermedad prolongada }(\mathrm{p}=.004) \\
\text { Enfermedad súbita }(\mathrm{p}=.025)\end{array}$ \\
\hline Edad Fallecido & $>65$ años $(\mathrm{p}=.010))$ \\
\hline Tiempo de muerte & $6-12$ meses $(p=.001)$ \\
\hline Vínculo & Padres $(p=.002)$ \\
\hline Tipo de PIC & $\begin{array}{l}\text { Consulta nueva }(\mathrm{p}=.006) \\
\text { Consulta nueva preferente }(\mathrm{p}=.015)\end{array}$ \\
\hline Derivación & A petición derivante $(\mathrm{p}=.001)$ \\
\hline Estado civil & $\begin{array}{l}\text { Soltero }(\mathrm{p}=.017) \\
\text { Casado }(\mathrm{p}=.024) \\
\text { Viudos }(\mathrm{p}=.027)\end{array}$ \\
\hline Nivel educativo & EGB y FP1 $(\mathrm{p}=.018)$ \\
\hline Situación Laboral & $\begin{array}{l}\text { Activo }(\mathrm{p}=.006) \\
\text { Incapacidad laboral transitoria o permanente }(\mathrm{p}=.018)\end{array}$ \\
\hline Diagnóstico & Trastornos neuróticos $(\mathrm{p}=.004)$ \\
\hline Tipo de tratamiento & $\begin{array}{l}\text { Psiquiátrico }(\mathrm{p}=.004) \\
\text { Consulta psicoterapéutica }(\mathrm{p}=.027)\end{array}$ \\
\hline Tipo de mediación & $\begin{array}{l}\text { Ansiolítico }(\mathrm{p}=.046) \\
\text { Antidepresivos }+ \text { Ansiolíticos }(\mathrm{p}=.002)\end{array}$ \\
\hline Tiempo tratamiento individual & $\begin{array}{l}7-9 \operatorname{citas}(\mathrm{p}=.042 \\
>10 \operatorname{citas}(\mathrm{p}=.012)\end{array}$ \\
\hline
\end{tabular}


Gráfica 2

Medias y Desviaciones Típicas de las puntuaciones

de la Escala de Prigerson antes y después del tratamiento.

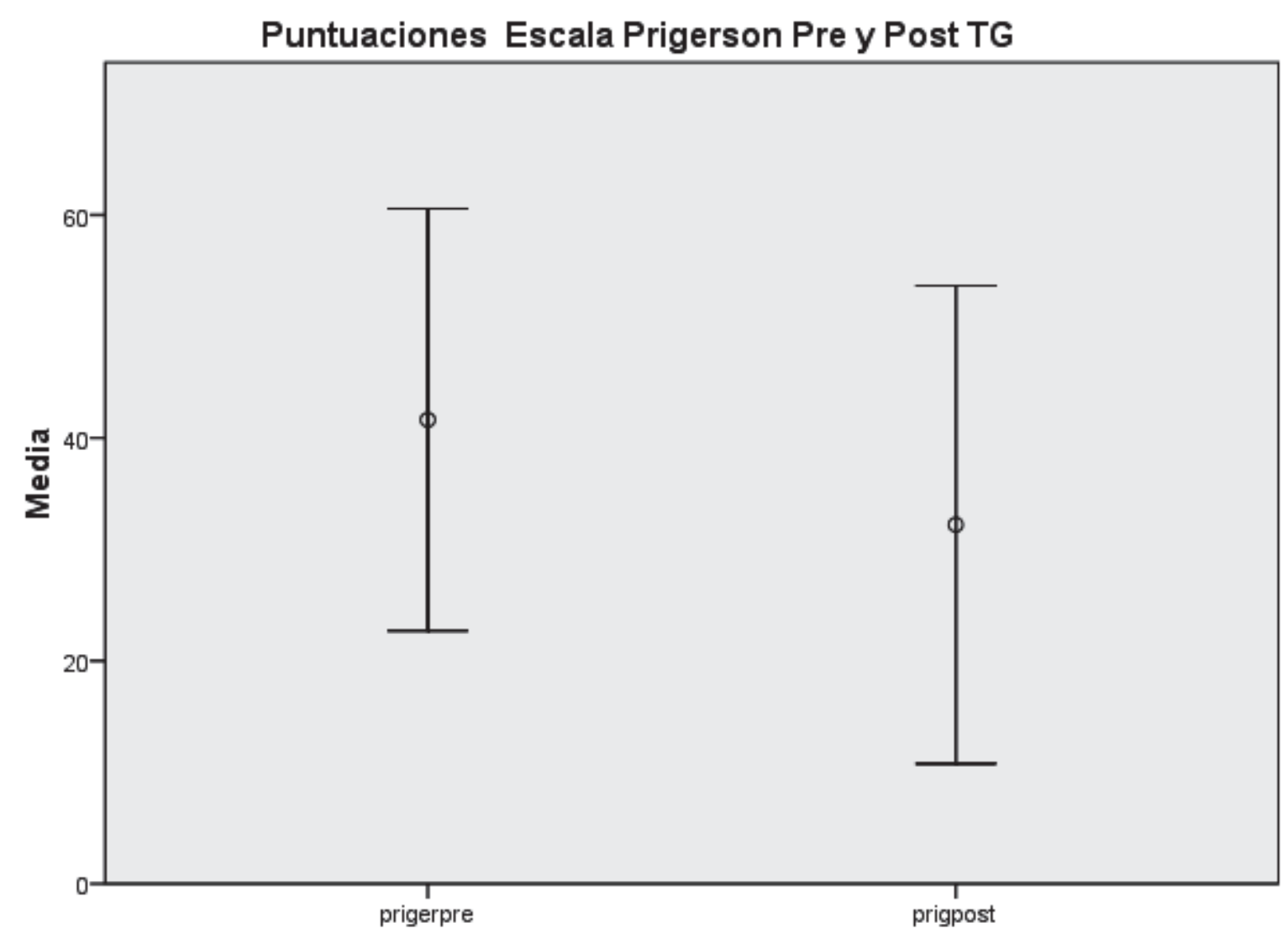

Barras de error: +/. 2 DT

\section{Discusión y Conclusiones}

Las puntuaciones de la Escala de Prigerson varían significativamente para la mayoría de los sujetos que han intervenido en las Terapias de Grupo (TG). La media de las puntuaciones antes de la TG es de 40,63 (DT=9,46) y disminuye a 32,21 (DT=10,71) una vez finalizado el grupo. Al final del tratamiento 6 sujetos $(25 \%)$ puntúan por debajo de punto de corte para el Duelo patológico en la Escala de Prigerson, que expresa un duelo dentro de la variación de lo normal en estos sujetos.

En la comparación de las variables descriptivas sociolaborales y familiares de los pacientes pre y post TG, encontramos que los sujetos con estudios de EGB y FP1 aparecen diferencias significativas en las comparaciones de las puntuaciones de la Escala de Prigerson antes y después del tratamiento grupal: mejoran más en la 
ORIGINALES Y REVISIONES

elaboración del duelo que otros con estudios de nivel superior o los que no tienen estudios primarios. Del mismo modo tanto las personas casadas como los viudos o solteros mejoran de la evolución del duelo, sin que exista diferencias en el estado civil. La situación laboral interviene positivamente tanto para las personas activas como aquellos que se encuentran con incapacidad laboral transitoria o permanente.

Entre las variables Clínicas y de Tratamiento, los pacientes con diagnóstico de Estructura neurótica mejoran más que los que presentan otras patologías como los trastornos de personalidad o trastornos afectivos. Los pacientes con tratamientos largos en Salud Mental (7-9 citas y >10 citas) se benefician de forma más eficaz de la terapia grupal, así como aquellos que han recibido tratamiento exclusivamente psiquiátrico con fármacos de tipo combinado (antidepresivos y ansiolíticos).

Las circunstancias de la muerte también interviene en la evolución del DC de los pacientes: Los sujetos que han perdido a un familiar por enfermedad súbita o prolongada puntúan significativamente mejor en la evolución del duelo que aquellos que han fallecido por otras causas (suicidio o accidentes). La evolución positiva en la elaboración del duelo es mayor en aquellos deudos que han perdido a uno de sus padres que aquellos que han perdido a una pareja, hijo o hermano y si ha trascurrido entre 6-12 meses entre el fallecimiento y el inicio del Grupo terapéutico.

Entre los factores terapéuticos definidos por Bloch y cols. (8) como "el proceso que ocurre en terapia de grupo por la intervención del terapeuta, los otros miembros del grupo y el propio paciente, y que contribuye a la mejoría de éste" que pueden ayudar a la mejoría y elaboración del duelo complicado en nuestro marco terapéutico grupal señalamos como los más relevantes los siguientes por lo que los contenidos de las sesiones se han dirigido a estimular dichos factores:
1. Altruismo (+)
2. Cohesión grupo $(+)$
3. Universalidad $(+)$
4. Aprendizaje interpersonal -Interiorización- $(++)$
5. Aprendizaje interpersonal -Exteriorización- $(++)$
6. Orientación $(+)$
7. Verbalización $(+++)$
8. Emulación (Identificación) (++)
9. Reactivación de la experiencia familiar $(+)$
10. Comprensión de sí mismo (+)
11. Estimulación recíproca $(++)$
12. Conciencia de la propia realidad $(++)$
(+=A veces; ++=Mucho; +++=Siempre).

De este modo y aunque tampoco es objetivo explícito de esta investigación el análisis de los factores terapéuticos observamos que la verbalización es el más rele- 
vante, junto con el aprendizaje interpersonal, la identificación entre todos los compañeros del grupo y la estimulación recíproca.

Aunque no es nuestro propósito en esta investigación analizar los contenidos de las sesiones y la evolución de los mismos a lo largo del proceso psicoterapéutico, los temas tratados son semejantes a los analizados por otros autores como Worden (5), Niemeyer (17) y Pérez Salas y cols. (16). Los contenidos de las sesiones de uno de los grupos de tratamiento los hemos resumido a continuación:

(Sesión 1). Relación con el fallecido. Sobre la despedida. ¿Qué hay detrás de la muerte? Formas de padecer el dolor. Enfado y culpa. No soportar lo que rodea a la muerte

(Sesión 2). Qué hacer con los objetos de vinculación. Enfado y culpa. Vínculo con el fallecido. Aceptar la ausencia. Tiempo para elaborar la pérdida . Necesidad de estar solo

(Sesión 3) No aceptación muerte. Culpa por ser feliz. Tiempo necesario para elaborar el duelo. Soledad y Compañía. Culpa por no cumplir deseos del fallecido. Vacío, soledad y confusión. Estados de ánimo variables. Se puede ser uno mismo aunque falte el otro. Lo que se lleva quién fallece.

(Sesión 4) No aceptación. Sobre el duelo múltiple. Tiempo del duelo. Recuerdos malos. Tipo de recuerdos. Protesta. "Nunca se esta preparado para morir" . No saber qué hacer sin el Otro. Impotencia ante enfermedad mortal. Vivir sin el fallecido. Sobre la despedida.

(Sesión 5) Búsqueda de afecto en los otros. La vida ya no es igual. Ocultación de sentimientos. ¿Qué hacer con los recuerdos?. Aislamiento versus vida social. Tiempo para pensar. Aceptación. El antes y después de la muerte. La muerte no se supera. "Reaprender a llevarla". Vivir y recordar. La expresión de sentimientos no es mala.

(Sesión 6) Sentimiento vacío. Vivir y pensar en el fallecido. No sentirse entendido. Los vivos no reemplazan a los muertos. No aceptación produce daño. Aprender a vivir sin el Otro. Vacío. Reactivación depresiones previas. Recolocar los sentimientos. Recuerdo y tristeza versus alegría.

(Sesión 7) Sobre ir al cementerio. Ponerse en el lugar del fallecido. Ambivalencia ante la terapia. "La muerte es inhumana". Rescatar el presente. "Los muertos son el pasado". Lo que se tiene se puede perder.

(Sesión 8) Miedo a mejorar. Tiempo para elaborar el Dolor. Impotencia para controlar el dolor. Dolor por la ausencia. Necesidad de formar parte de un grupo "que te entienda". "Ya no somos los mismos". El duelo como un proceso. Afecto a otros. Cuidar a los vivos. "La muerte es injusta".

(Sesión 9). Las creencias ayudan a superar el duelo. Aprender que la muerte no se puede evitar. Papel de lo religioso. La pena por la ausencia continúa. Expresión de sentimientos con las personas cercanas. Sobre las fotos y pertenencias. Recordar sin amargura y sin temor. Forma personal de elaborar la pérdida. 
ORIGINALES Y REVISIONES

(Sesión 10) Duelo por la finalización del grupo. Necesidad de formar parte de un grupo (fuera). Relación previa fallecido/dolor actual. El duelo: proceso individual. Derecho a la felicidad. Integrar la pérdida sin asustarse ni deprimirse. Despedidas. Recordar sin deprimirse. ¿Cómo se puede ayudar a otros?.

Los contenidos se repiten a lo largo de las sesiones, van y vienen, pero nuestro objetivo siempre es el mismo: tratamos que los sujetos integren los mismos dentro de un proceso personal en el proceso grupal que devuelve los mismos de forma ordenada, diferenciada y elaborada.

Comparando los resultados a través de la Escala de Prigerson entre los pacientes antes y después del tratamiento podemos afirmar que la terapia grupal de tiempo limitado a 10 sesiones es eficaz tal y como la estructuramos en nuestro modelo psicoterapéutico. De los 8 trabajos revisados por Kato y Mann (6), solo uno de los estudios refleja resultados positivos y en 6 de ellos la intervención grupal no tiene ningún efecto y en otro solo se encuentran resultados levemente positivos. A diferencia de nuestra muestra compuesta por pacientes con DC, los sujetos de los grupos revisados en dicho metanálisis no son población clínica con un diagnostico claro de Duelo complicado y están constituidos por sujetos en duelo con el objetivo de buscar apoyo y promover actividades de índole social sin cambios cognitivos y/o reestructurantes de la sintomatología asociada al duelo, mientras nuestros grupos son eminentemente clínicos con objetivos psicoterapéuticos claros y definidos.

Compartimos con estudios en nuestro entorno los resultados en cuanto a: 1) Elaboración de la pérdida y la adaptación a la nueva situación (10); 2) Mejoría en aspectos depresivos, mecanismos defensivos, relaciones interpersonales (11). Podemos afirmar que la mejora de los síntomas relacionados con el duelo patológico es mayoritaria y generalizada en nuestros pacientes, aunque la medida que adoptamos para evaluar dicho cambio es el Test de Prigerson (1) que pivota sobre el conjunto de síntomas que dan lugar al duelo patológico centradas en malestar por la pérdida (pensamientos intrusivos sobre la persona fallecida, añoranza, búsqueda del fallecido y soledad como resultado del fallecimiento) y síntomas de "malestar traumático" (falta de metas y/o inutilidad respecto al futuro, sensación subjetiva de indiferencia o ausencia de respuesta emocional, dificultades para aceptar la muerte, excesiva irritabilidad, amargura y/o enfado en relación a la muerte).

Al finalizar el tratamiento grupal el 58,3\% de los sujetos solicitan el alta en Salud Mental y el 37,5\% continúan recibiendo asistencia psiquiátrica y/o psicoterapéutica o tratamiento combinado farmacológico y psicoterapéutico. En todo caso es conveniente un seguimiento de los pacientes y una evaluación clínica en un periodo de 2-5 años una vez finalizada la terapia de grupo para estudiar la estabilidad de los cambios producidos en la terapia grupal en relación a la mejoría de los síntomas asociados a la elaboración complicada de los duelos. 
ORIGINALES Y REVISIONES

\section{BIBLIOGRAFÍA:}

(1) Prigerson, HG; Maciejewski, P K; Reynolds, Ch F.; Bierhals, A. J.; Newson, J. T. y cols. Inventory of Complicated Grief: A scale to measure maladaptive symptoms of loss. Psychiatry Res (1995), 59: 65-79.

(2) Olmeda, Ma Soledad; García, Ana María. Parentesco y duelo. Revista electrónica Interpsiquis (2006). Disponible en: http://www.psiquiatria.com/articulos/depresion/24840/.

(3) Gil-Juliá, B.; Bellver, A.; Ballester, R. Duelo: Evaluación, Diagnóstico y Tratamiento". Psicooncología (2008), Vol 5 nº 1: 103-116.

(4) Tizón, J.L. Pérdida, Pena, Duelo. 2004. Barcelona. Paidós.

(5) Worden JW. El Tratamiento del duelo: asesoramiento psicológico y terapia. 1997. Barcelona. Paidós.

(6) Kato PM; Mann, T.A.. Synthesis of psychological interventions for the bereaved. Clin Psychol Rev (1999). 19 (3): 275-296.

(7) Yalom, ID. Teoría y práctica de la psicoterapia de grupo. 1986. México. FCE (1 $1^{\mathrm{a}}$ ed.).

(8) Bloch, S.; Crouch, E.; ReIBSTEIN, J. Therapeutic factors in group psychotherapy. Arch Gen Psy (1981), 38: 519-526.

(9) Almenta, E., García Robles, Ma P., Gónzález Duro, E. Factores terapéuticos en dinámica grupal. Rev Asoc Esp Neuropsiq (1994), Vol XIV, nº 47-48: 83-97.

(10) Lacaste, MA, KroefF, P.; Sastre, P. Casero, A.; Martínez, AM. Una intervención en grupo para personas en duelo. Med Paliat (2000), Vol 7, $\mathrm{n}^{\circ}$ 1: 6-9.

(11) Forteza, Ma Antonia. ¿Es útil la psicoterapia grupal en los procesos de duelo?. Clin Anal Grup (2003), Vol 25(1): 93-99.

(12) García-García, J.A.; Landa, V.; Trigueros, M.C.; Calvo, P.; Gaminde, I. El duelo por la pérdida del cónyuge: un estudio mediante grupos de discusión en Atención Primaria. Aten Primaria (1996), Vol 18, n 19.

(13) Novellas, A.; Bleda, M. Grupos de poyo al proceso de duelo: 5 años de evolución. Med Paliat (2005), Vol 12, $\mathrm{n}^{\mathrm{o}}$ 1: 17-23.

(14) JaRnÉ, A.; GarCía, A.M.; ToRres, S. Asesoramiento grupal en duelo traumático. An Psiquiatr (2006), Vol 21, nº 5: 230-236.

(15) Piper, WE.; Ogrodniezuk, JS.; Weideman, R.; Joyce, AS.; Roie, JS. Group Composition and Group Therapy for Complicated Grief. J Consul Clin Psy (2007), Vol. 75: 116-125.

(16) Pérez Sales, P.; HernánGoméz, L. Terapia en duelo complicado: un enfoque desde los valores de cambio positive. Rev. Psiquiat Publica (2000), Dic, Vol 12, n 3:273-284.

(17) NiemEYer, R.A. Aprender de la pérdida. Una Guía para afrontar el duelo. 2002. Barcelona. Paidós. 


\section{ESCALA DE PRIGERSON}

Por favor, ponga una cruz en la opcion que mejor describa su experiencia tras la muerte de su familiar.

\begin{tabular}{|l|l|l|l|l|l|}
\cline { 2 - 7 } \multicolumn{1}{l|}{} & SIEMPRE & $\begin{array}{r}\text { A } \\
\text { MENUDO }\end{array}$ & $\begin{array}{c}\text { ALGUNAS } \\
\text { VECES }\end{array}$ & $\begin{array}{l}\text { RARAS } \\
\text { VECES }\end{array}$ & NUNCA \\
\hline $\begin{array}{l}\text { fallecido, que me resulta dificil hacer } \\
\text { las cosas como las hacia normalmente. }\end{array}$ & & & & & \\
\hline $\begin{array}{l}\text { 2. Los secuerdos de la persona que murió } \\
\text { me trastoman }\end{array}$ & & & & & \\
\hline $\begin{array}{l}\text { 3. Siento que no puedo aceptar la muerte } \\
\text { de la persona fallecida }\end{array}$ & & & & & \\
\hline $\begin{array}{l}\text { 4. Anhelo a la persona que murio } \\
\text { 5. Me siento atraido por los lugares y las } \\
\text { cosas relacionadas con la persona } \\
\text { fallecida }\end{array}$ & & & & & \\
\hline
\end{tabular}

\begin{tabular}{|l|l|l|l|l|l|}
\hline $\begin{array}{l}\text { 6. No puedo evitar sentirme enfadado } \\
\text { con su muerte }\end{array}$ & & & & & \\
\hline 7. No me puedo creer que haya sucedido & & & & & \\
\hline 8. Me siento aturdido por lo sucedido & & & & & \\
\hline $\begin{array}{l}\text { 9. Desde que élella murió me resulta dificil } \\
\text { confiar en la gente }\end{array}$ & & & & & \\
\hline
\end{tabular}

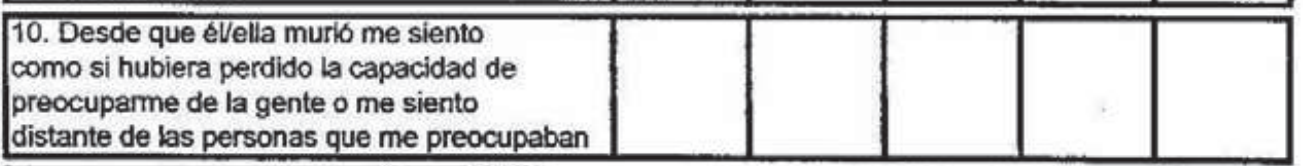

\begin{tabular}{|l|l|l|l|l|l|}
\hline $\begin{array}{l}\text { 11. Me siento solo/a la mayor parte del } \\
\text { tiempo desde que éVella ha muerto }\end{array}$ & & & & & \\
\hline
\end{tabular}

\begin{tabular}{|l|l|l|l|l|l|}
\hline $\begin{array}{l}\text { 12. Siento dolores en la misma zona del } \\
\text { cuerpo o tengo algunos de los sintomas } \\
\text { que sufrla la persona que murio }\end{array}$ & & & & & \\
\hline
\end{tabular}

\begin{tabular}{|l|l|l|l|l|l|}
\hline $\begin{array}{l}\text { 13. Me tomo la molestia de desviarme de } \\
\text { mi camino para evitar los recuerdos de la } \\
\text { persona que murió }\end{array}$ & & & & & \\
\hline
\end{tabular}

\begin{tabular}{|l|l|l|l|l|l|}
\hline $\begin{array}{l}\text { 14. Siento que la vida esta vacla sin la } \\
\text { persona que murio }\end{array}$ & & & & & \\
\hline
\end{tabular}

\begin{tabular}{|l|l|l|l|l|l|}
\hline $\begin{array}{l}\text { 15. Escucho la voz de la persona } \\
\text { fallecida hablándome }\end{array}$ & & & & & \\
\hline $\begin{array}{l}\text { 16. Veo a la persona que murio de pie } \\
\text { delante de mi }\end{array}$ & & & & & \\
\hline $\begin{array}{l}\text { 17. Slento que es injusto que yo viva } \\
\text { mientras elvella ha muerto }\end{array}$ & & & & & \\
\hline $\begin{array}{l}\text { 18. Siento amargura por la muerte de } \\
\text { esepensona }\end{array}$ & \begin{tabular}{|l|l|l|l|l|l|}
\hline $\begin{array}{l}\text { 19. Siento envidia de otras personas que } \\
\text { no han perdido a nadie cercano }\end{array}$ & & & & & \\
\hline
\end{tabular}
\end{tabular}

